\title{
Tolerância do girassol a herbicidas aplicados em pós-emergência ${ }^{1}$
}

\section{Tolerance of sunflower to herbicide application in post-emergence}

\section{Ronaldo Matias Reis ${ }^{2}$; Matheus Freitas $\mathrm{Souza}^{3}$; Guilherme Pereira Queiroz ${ }^{3}$; Isadora Garcia} Siebert ${ }^{4}$; Daniel Valadão Silva ${ }^{5}$; Evander Alves Fereira ${ }^{6}$; Antônio Alberto Silva ${ }^{7}$

Resumo - Para avaliar a tolerância do girassol à aplicação de herbicidas em pós-emergência foi realizado um experimento em casa de vegetação em delineamento inteiramente ao acaso, com cinco repetições. Os tratamentos foram constituídos da aplicação de flumioxazin, fomesafen, lactofen, fluazifop-p-buthyl, fluazifop-p-buthyl + fomesafen, fenoxaprop-p-ethyl + clethodim, haloxyfop-pmethyl, clethodim, chlorimuron-ethyl e isoxaflutole aos 22 dias após a emergência do girassol, além de uma testemunha sem aplicação. Avaliou-se a intoxicação visual das plantas, a altura de plantas, o número de folhas, a área foliar, a matéria seca total e as seguintes variáveis fisiológicas: taxa de assimilação líquida de $\mathrm{CO}_{2}$ (A), condutância estomática de vapor d'água (gs) taxa transpiratória $(E)$, relação entre as concentrações interna e externa de $\mathrm{CO}_{2}\left(\mathrm{C}_{\mathrm{i}}: \mathrm{C}_{\mathrm{a}}\right)$, eficiência instantânea no uso da água (EUA) e consumo de $\mathrm{CO}_{2}(\Delta \mathrm{C})$. A resposta do girassol foi dependente do herbicida aplicado. Flumioxazin, fenoxaprop-p-ethyl + clethodim, haloxyfop-p-methyl, fluazifop-p-buthyl e clethodim promoveram as menores intoxicações ao girassol, de maneira que a fisiologia e o crescimento das plantas não foram prejudicados. Ao avaliar as características fisiológicas, o fomesafen, a mistura fluazifop-p-buthyl + fomesafen e chlorimuron-ethyl destacamse como os mais danosos à cultura, afetando negativamente as variáveis $\mathrm{A}$, gs, $\mathrm{E}$ e $\Delta \mathrm{C}$ das plantas tratadas com esses produtos.

Palavras-chaves: Helianthus annuus, fotossíntese, condutância estomática, análise de crescimento

\begin{abstract}
To evaluate the Sunflower tolerance to herbicide application in post-emergence was conducted a experiment in greenhouse with design in completely randomized, with five repetitions. The treatments consisted of the application of flumioxazin, fomesafen, lactofen, fluazifop-pbuthyl, fluazifop-p-buthyl + fomesafen, fenoxaprop-p-ethyl + clethodim, haloxyfop-P-methyl, clethodim, chlorimuron-ethyl and isoxaflutole at 22 days after emergence sunflower, and a control without application. We evaluated the visual intoxication of plants, plant height, leaf number, leaf area, total dry matter and the following physiological variables: net assimilation rate of CO2 (A), stomatal conductance vapor water ( $\mathrm{gs}$ ) transpiration rate $(\mathrm{E})$, relation between internal and external concentrations of $\mathrm{CO} 2(\mathrm{Ci} \mathrm{Ca})$, instantaneous water use efficiency (US) and $\mathrm{CO} 2$ consumption

\footnotetext{
${ }^{1}$ Recebido para publicação em 28/05/2014 e aceito em 31/05/2015.

${ }^{2}$ Mestre em Fitotecnia - Universidade Federal de Viçosa. Viçosa, Minas Gerais, Brasil. ronaldo.reis@ufv.br (*autor para correspondência).

${ }^{3}$ Mestrando em Fitotecnia - Universidade Federal de Viçosa. Viçosa, Minas Gerais, Brasil.

${ }^{4}$ Aluna de graduação - Universidade Federal de Viçosa. Viçosa, Minas Gerais, Brasil

${ }^{5}$ Professor do Departamento de Fitotecnia - Universidade Federal Rural do Semi-Árido, Mossoró, Rio Grande do Norte, Brasil.

${ }^{6}$ Pós-doutorando em Fitotecnia - Universidade Federal Vales do Jequitinhonha e Mucuri. Diamantina, Minas Gerais, Brasil.

${ }^{7}$ Professor do Departamento de Fitotecnia - Universidade Federal de Viçosa. Viçosa, Minas Gerais, Brasil.
} 
$(\Delta C)$. Sunflower response was dependent on the applied herbicide. Flumioxazin, fenoxaprop-pethyl + clethodim, haloxyfop-P-methyl, fluazifop-p-buthyl and clethodim promoted smaller sunflower intoxication, so that the physiology and plant growth were not affected. In assessing the physiological characteristics, the fomesafen, fluazifop-p-buthyl + fomesafen mixture and chlorimuron-ethyl were most damaging to the culture, negatively affecting the variables A, gs, E and $\Delta \mathrm{C}$ of plants treated with these products.

Keywords: Helianthus annuus, photosynthesis, stomatal conductance, growth analysis

\section{Introdução}

O girassol (Helianthus annuus L.) é uma das quatro espécies oleaginosas mais cultivada no mundo, sendo utilizada na alimentação humana e animal, como também na produção de biocombustíveis (Brighenti, 2012). A espécie apresenta grande capacidade de adaptação a diferentes condições ambientais, por isso é cultivada em todos os continentes (Castro et al., 1997). No Brasil, a cultura vem ganhando espaço, principalmente, nas regiões de Cerrado, como opção para o cultivo na safrinha.

Um dos principais problemas relacionados ao cultivo do girassol é a interferência exercida pelas plantas daninhas. Estas competem com o girassol por recursos de crescimento, causando sérios danos à cultura, tais como redução do porte, diminuição da área foliar, do diâmetro do caule e do capítulo (Brighenti, 2012). O arranjo de plantas adotado nos cultivos comerciais, com espaçamento entre linhas de 70 a $90 \mathrm{~cm}$ e com baixa densidade de plantas comparada a outras culturas, permite que o solo fique descoberto por um longo período favorecendo a captação de radiação solar pelas plantas daninhas e, consequentemente, favorecendo o crescimento destas espécies (Elezovic et al., 2012).

No controle de plantas daninhas, a ferramenta mais eficaz e viável economicamente é o método químico, porém, existem poucas opções de herbicidas registrados para o controle das plantas daninhas em girassol. Na sua maioria são herbicidas préemergentes e com ação principalmente sobre gramíneas, como por exemplo, alachlor e trifluralin (Brasil, 2014). Além disso, o girassol é bastante sensível, sobretudo a herbicidas pós- emergentes que controlam espécies daninhas dicotiledôneas (Brighenti et al., 2003).

De modo geral, os herbicidas inibidores da ACCase (acetilcoenzima-A-carboxilase) são seletivos para o girassol e os grupos de herbicidas das triazinas e das imidazolinonas apresentam alta toxidade à cultura em pósemergência (Castro et al., 1997). No entanto, não há informações consistentes da tolerância do girassol a outros herbicidas aplicados em pós-emergência. No trabalho de Pannacci et al. (2007), os herbicidas aclonifen, oxyfluorfen, aclonifen + quizalofop-ethyl aplicados em pósemergência do girassol (estádio V2-V4) apresentaram baixa toxicidade a cultura e controle eficiente das espécies dicotiledôneas, sendo que a mistura (aclonifen+quizalofopethyl) apresentou maior espectro de ação no controle das plantas daninhas.

A ação dos herbicidas, de algum modo afeta o metabolismo das plantas, mesmo nas doses recomendadas. Clorose e necroses foliares, redução do crescimento das plantas são sintomas de intoxicação evidenciados após aplicação dos herbicidas (Zablotowicz e Reddy, 2007). Estes sintomas podem ser a causa ou mesmo consequência de alterações no metabolismo fotossintético. Estudos da fisiologia do girassol, principalmente associados às trocas gasosas e a eficiência na utilização radiação luminosa, que são processos básicos na produção de biomassa, podem indicar os danos provocados pelos herbicidas às plantas de girassol.

Neste sentido, objetivou-se nesta pesquisa avaliar a tolerância do girassol à diferentes herbicidas aplicados em pósemergência, bem como, avaliar o efeito desses 
produtos nas características fisiológicas das plantas.

\section{Material e Métodos}

O experimento foi realizado em casa de vegetação no Departamento de Fitotecnia da Universidade Federal de Viçosa, em Viçosa MG, durante o período de abril a junho de 2013. O solo utilizado foi um Latossolo VermelhoAmarelo Distrófico, com textura argilosa (46\% de argila, $15 \%$ de silte e $39 \%$ de areia) cujas principais características químicas encontramse na Tabela 1. Para a correção e adubação do solo foram aplicados $2000 \mathrm{~kg} \mathrm{ha}^{-1}$ de calcário calcítico, $350 \mathrm{~kg} \mathrm{ha}^{-1}$ de superfosfato simples, $100 \mathrm{~kg} \mathrm{ha}^{-1}$ de sulfato de amônia e $120 \mathrm{~kg} \mathrm{ha}^{-1}$ de cloreto de potássio. $\mathrm{Na}$ adubação de cobertura foram utilizados $200 \mathrm{~kg} \mathrm{ha} \mathrm{ho}^{-1} \mathrm{do}$ formulado NPK 20-5-20, aplicados 20 dias após a emergência (DAE) das plantas.

O delineamento experimental utilizado foi o inteiramente casualizado, com cinco repetições. Os tratamentos foram constituídos da aplicação do flumioxazin, fomesafen, lactofen, fluazifop-p-buthyl, fluazifop-p-buthyl + fomesafen, fenoxaprop-p-ethyl + clethodim, haloxyfop-p-methyl, clethodim, chlorimuronethyl e isoxaflutole aos 22 DAE, além de uma testemunha sem aplicação (Tabela 2).

Tabela 1. Caracterização química do solo. Viçosa (MG), 2013.

\begin{tabular}{|c|c|c|c|c|c|c|c|c|c|c|c|c|}
\hline $\mathrm{pH}$ & $\begin{array}{l}\mathrm{P} \\
\mathrm{mgdn}\end{array}$ & & $\mathrm{Ca}^{+2}$ & $\mathrm{Mg}^{+2}$ & $\begin{array}{l}\mathrm{Al}^{+3} \\
--\mathrm{cm}\end{array}$ & $\begin{array}{l}\mathrm{H}+\mathrm{Al} \\
\mathrm{lm}^{-3}-\cdots\end{array}$ & $\mathrm{t}$ & $\mathrm{T}$ & & $\mathrm{m}$ & $\begin{array}{c}\mathrm{MO} \\
\text { dagkg }^{-1}\end{array}$ & $\begin{array}{l}\text { P-rem } \\
\text { mgL }^{-1}\end{array}$ \\
\hline 4,2 & 1,1 & 30 & 0,8 & 0,5 & 0,6 & 6,27 & 1,98 & 7,65 & 18 & 30 & 2,7 & 22,7 \\
\hline
\end{tabular}

Extratores: $\mathrm{pH}$ - $\mathrm{H}_{2} \mathrm{O}$; P e K - Mehlich 1; Ca, Mg, $\mathrm{Al}$ - $\mathrm{KCl} 1 \mathrm{~mol} \mathrm{~L}^{-1} ; \mathrm{H}+\mathrm{Al}$ - Ca(OAc) $)_{2} 0,5 \mathrm{~mol} \mathrm{~L}^{-1}$.

Tabela 2. Características dos herbicidas aplicados em pós-emergência de plantas de girassol. Viçosa (MG), 2013.

\begin{tabular}{|c|c|c|c|}
\hline Mecanismo de ação ${ }^{1}$ & Nome comum & $\begin{array}{c}\text { Produto } \\
\text { Comercial }\end{array}$ & $\begin{array}{c}\text { Dose i.a. } \\
\left(\mathrm{g} \mathrm{ha}^{-1}\right)\end{array}$ \\
\hline \multirow{3}{*}{ Inibidores da PPO } & Flumioxazin & Sumisoya ${ }^{\circledR}$ & 50 \\
\hline & Fomesafen & Flex $^{\circledR}$ & 250 \\
\hline & Lactofen & Cobra $^{\circledR}$ & 180 \\
\hline \multirow{2}{*}{ Inibidor da ACCase e PPO } & Fluazifop-p-buthyl + fomesafen & Fusiflex $^{\circledR}$ & $225+225$ \\
\hline & Fenoxaprop-p-ethyl + clethodim & Podium $S^{\circledR}$ & $93,75+93,75$ \\
\hline \multirow{3}{*}{ Inibidores da ACCase } & Haloxyfop-p-methyl & Verdict $^{\circledR}$ & 60 \\
\hline & Fluazifop-p-buthyl & Fusilade $^{\circledR}$ & 187,5 \\
\hline & Clethodim & Select $240 \mathrm{EC}^{\circledR}$ & 96 \\
\hline Inibidores da ALS & Chlorimuron-ethyl & Classic $^{\circledR}$ & 20 \\
\hline Despigmentadores & Isoxaflutole & Provence $^{\circledR}$ & 75 \\
\hline
\end{tabular}

Foram semeadas quatro sementes de girassol, hibrido HELIO 250, em vasos com capacidade volumétrica de $5 \mathrm{dm}^{3}$, sendo o desbaste realizado aos 10 dias após o semeio e 5 dias após a emergência, deixando-se uma planta por unidade experimental. A aplicação dos herbicidas foi realizada aos 22 DAE das plantas com pulverizador costal pressurizado a $\mathrm{CO}_{2}$, operando à pressão constante de $3,0 \mathrm{kgf} \mathrm{cm}^{-2}$, equipado com barra de duas pontas TT 110.02 espaçadas de $50 \mathrm{~cm}$, a uma altura de aproximadamente $40 \mathrm{~cm}$ do alvo, e volume de calda equivalente a $150 \mathrm{~L} \mathrm{ha}^{-1}$.

Aos 5, 10, 15, 20 e 25 dias após a aplicação dos herbicidas avaliou-se a intoxicação visual das plantas de girassol através de escala de notas na qual $0 \%$ representa nenhuma injúria e $100 \%$ morte das plantas, conforme a metodologia da SBCPD (1995). 
Aos 25 dias após a aplicação dos herbicidas foram analisadas as trocas gasosas das plantas de girassol, sendo as avaliações realizadas entre 09:00 e 10:00 h, na terceira folha completamente expandida a partir do ápice das plantas. As taxas de assimilação líquida de $\mathrm{CO}_{2}(A)$, a condutância estomática $\left(g_{\mathrm{s}}\right)$, a taxa transpiratória $(E)$, a razão entre as concentrações interna e externa de $\mathrm{CO}_{2}\left(C_{\mathrm{i}}: C_{\mathrm{a}}\right)$, a eficiência instantânea no uso da água (EUA) e o consumo de $\mathrm{CO}_{2}(\Delta \mathrm{C})$ foram determinadas em sistema aberto, sob luz saturante artificial (1.500 $\mu$ mol fótons $\mathrm{m}^{-2} \mathrm{~s}^{-1}$ ), concentração de $\mathrm{CO}_{2}$ ambiente, com um analisador de gás a infravermelho portátil (LICOR 6400XT, LiCOR, Lincoln, EUA).

Aos 32 dias após a aplicação foram determinadas a altura, número de folhas e área foliar do girassol. Além disso, todo o material vegetal foi colhido e, posteriormente, seco em estufa com circulação forçada de ar, a $65^{\circ} \mathrm{C}$, até atingir peso constante para determinação da matéria seca.

Os dados obtidos foram submetidos à análise de variância pelo teste F. Para a comparação das médias utilizou-se o teste de Scott-Knott, ao nível de 5\% de probabilidade.

\section{Resultados e Discussão}

Os herbicidas fomesafen, lactofen, fluazifop-p-buthyl + fomesafen e chlorimuronethyl foram os produtos que promoveram os maiores índices de intoxicação visual nas plantas de girassol em todas as avaliações realizadas (Tabela 3). O isoxaflutole promoveu intoxicação de $16 \%$ aos 5 DAA até $33 \%$ aos 25 DAA. Os herbicidas inibidores da enzima Acetil Coenzima A (fenoxaprop-p-ethyl + clethodim, haloxyfop-p-methyl, fluazifop-p-buthyl e clethodin), reconhecidamente graminicidas, promoveram baixa intoxicação das plantas.

Tabela 3. Intoxicação de plantas de girassol aos 5, 10, 15, 20 e 25 dias após a aplicação dos herbicidas. Viçosa (MG), 2013.

\begin{tabular}{|c|c|c|c|c|c|c|}
\hline \multirow{2}{*}{ Tratamentos } & \multirow{2}{*}{ Dose (g i.a. ha $\left.{ }^{-1}\right)$} & \multicolumn{5}{|c|}{ Fitointoxicação (\%) } \\
\hline & & $5 \mathrm{DAA}$ & 10 DAA & $15 \mathrm{DAA}$ & 20 DAA & $25 \mathrm{DAA}$ \\
\hline Testemunha $^{1}$ & $-\overline{---}$ & $0 \mathrm{a}$ & $0 \mathrm{a}$ & $0 \mathrm{a}$ & $0 \mathrm{a}$ & $0 \mathrm{a}$ \\
\hline Flumioxazin & 50 & $11 \mathrm{c}$ & $12 \mathrm{c}$ & $10 \mathrm{c}$ & $10 \mathrm{~b}$ & $7 \mathrm{~b}$ \\
\hline Fomesafen & 250 & $70 \mathrm{e}$ & $72 \mathrm{e}$ & $78 \mathrm{f}$ & $73 \mathrm{~d}$ & $75 \mathrm{~d}$ \\
\hline Lactofen & 180 & $74 \mathrm{e}$ & $80 \mathrm{e}$ & $75 \mathrm{f}$ & $71 \mathrm{~d}$ & $76 \mathrm{~d}$ \\
\hline Fluazifop-p-buthyl + fomesafen & $225+225$ & $76 \mathrm{e}$ & $79 \mathrm{e}$ & $86 \mathrm{f}$ & $84 \mathrm{~d}$ & $87 \mathrm{~d}$ \\
\hline Fenoxaprop-p-ethyl + clethodim & $93,75+93,75$ & $8 \mathrm{~b}$ & $10 \mathrm{c}$ & $8 \mathrm{c}$ & $7 \mathrm{~b}$ & $7 \mathrm{~b}$ \\
\hline Haloxyfop-p-methyl & 60 & $4 \mathrm{~b}$ & $7 \mathrm{c}$ & $3 \mathrm{~b}$ & $2 \mathrm{a}$ & $2 \mathrm{a}$ \\
\hline Fluazifop-p-butyl & 187,5 & $7 \mathrm{~b}$ & $8 \mathrm{c}$ & $5 \mathrm{~b}$ & $2 \mathrm{a}$ & $2 \mathrm{a}$ \\
\hline Clethodim & 96 & $1 \mathrm{a}$ & $3 \mathrm{~b}$ & $1 \mathrm{a}$ & $2 \mathrm{a}$ & $1 \mathrm{a}$ \\
\hline Chlorimuron-ethyl & 20 & $25 \mathrm{~d}$ & $66 \mathrm{e}$ & $59 \mathrm{e}$ & $66 \mathrm{~d}$ & $71 \mathrm{~d}$ \\
\hline Isoxaflutole & 75 & $16 \mathrm{c}$ & $30 \mathrm{~d}$ & $29 \mathrm{~d}$ & $30 \mathrm{c}$ & $33 \mathrm{c}$ \\
\hline $\mathrm{CV}(\%)$ & & 14,17 & 10,75 & 12,97 & 13,59 & 13,43 \\
\hline
\end{tabular}

Brighenti (2012) observou que o herbicida chlorimuron-ethyl não foi seletivo ao girassol, para os híbridos de girassol Tera 8003 e Tera 8011, apresentando altos percentuais de toxicidade à cultura. Vale ressaltar que esses híbridos eram resistentes aos inibidores da enzima acetolactatosintase (ALS) e mesmo assim foram poucos tolerantes ao chlorimuronethyl conforme observado no presente trabalho.
Estes resultados também foram verificados por Brighenti et al. (2004) que salientaram que embora o chlorimuron seja também um herbicida inibidor da enzima ALS, pertence ao grupo químico das sulfonilureias, apresentando menor seletividade do que aqueles pertencentes ao grupo químico das imidazolinonas.

A altura das plantas de girassol (ALT) não foi afetada pela aplicação dos herbicidas 
flumioxazin, fenoxaprop-p-ethyl + clethodim, haloxyfop-p-methyl, fluazifop-p-butyl e clethodim (Tabela 4). No entanto, os demais herbicidas afetaram a altura em valores que variaram de 28 até $72 \%$ para o isoxaflutole e chlorimuron, respectivamente. Em trabalho de Brighenti et al. (2002), os autores verificaram que a diminuição da altura foi acompanhada da redução do diâmetro do capítulo, do peso de mil aquênios e da produtividade do girassol.

$\mathrm{O}$ número de folhas (NF) foi afetado negativamente pelos herbicidas clethodim, chlorimuron-ethyl e isoxaflutole, sendo que para os demais produtos testados não houve diferença do NF em relação à testemunha isenta de herbicida (Tabela 3). Apesar de não afetar o NF observou-se que o lactofen, o fomesafen e a mistura fluazifop-p-butyl + fomesafen reduziram a área foliar (AF) do girassol. O fomesafen e lactofen são herbicidas que atuam inibindo a enzima protoporfirinogênio oxidase (PPO) na presença de luz e oxigênio resultando na peroxidação dos lipídeos da membrana celular (Silva et al., 2007). Resultados semelhantes com esses herbicidas foram observados por Silva et al. (2012) para a mandioca o que refletiu em menor crescimento da cultura.

A matéria seca da parte aérea (MSP) das plantas de girassol apresentou decréscimo superior a $75 \%$ após a aplicação dos herbicidas fomesafen, lactofen, fluazifop-p-buthyl + fomesafen, chlorimuron-ethyl (Tabela 4). O isoxaflutole reduziu em aproximadamente $40 \%$ a MSP, enquanto que os demais herbicidas não afetaram a variável.

Tabela 4. Altura de planta (ALT), número de folhas (NF), área foliar (AF) e matéria seca da parte áerea (MSP) de girassol (Helianthus annus L.) tratada com diferentes herbicidas. Viçosa (MG), 2013.

\begin{tabular}{|c|c|c|c|c|c|c|c|c|c|}
\hline Tratamentos & Dose (g i.a. ha-1) & $\begin{array}{r}\text { ALT } \\
\mathrm{cm}\end{array}$ & & $\begin{array}{l}\mathrm{NF} \\
\text { un }\end{array}$ & & $\begin{array}{l}\mathrm{AF} \\
\mathrm{cm}^{2}\end{array}$ & & $\begin{array}{r}\text { MSP } \\
\text { g/plan }\end{array}$ & \\
\hline Testemunha $^{1}$ & $\begin{array}{l}---- \\
--\end{array}$ & 101,0 & $\mathrm{a}$ & 24 & a & 2040 & a & 60,9 & $\bar{a}$ \\
\hline Flumioxazin & 50 & 92,5 & $\mathrm{a}$ & 27 & $\mathrm{a}$ & 2352 & $\mathrm{a}$ & 58,5 & $\mathrm{a}$ \\
\hline Fomesafen & 250 & 65,5 & $\mathrm{~b}$ & 26 & a & 847 & $\mathrm{~b}$ & 15,0 & $\mathrm{c}$ \\
\hline Lactofen & 180 & 45,0 & $\mathrm{c}$ & 24 & $\mathrm{a}$ & 531 & c & 9,6 & c \\
\hline Fluazifop-p-buthyl + fomesafen & $225+225$ & 41,8 & $\mathrm{c}$ & 24 & $\mathrm{a}$ & 472 & $\mathrm{c}$ & 8,1 & c \\
\hline Fenoxaprop-p-ethyl + clethodim & $93,75+93,75$ & 98,4 & $\mathrm{a}$ & 25 & $\mathrm{a}$ & 2285 & a & 69,3 & a \\
\hline Haloxyfop-p-methyl & 60 & 97,6 & $\mathrm{a}$ & 24 & a & 2228 & a & 59,0 & $\mathrm{a}$ \\
\hline Fluazifop-p-butyl & 187,5 & 84,9 & $\mathrm{a}$ & 26 & $\mathrm{a}$ & 2337 & $\mathrm{a}$ & 55,2 & a \\
\hline Clethodim & 96 & 88,0 & $\mathrm{a}$ & 23 & $\mathrm{~b}$ & 2156 & a & 55,6 & $\mathrm{a}$ \\
\hline Chlorimuron-ethyl & 20 & 28,0 & $\mathrm{c}$ & 20 & $\mathrm{~b}$ & 974 & b & 12,8 & c \\
\hline Isoxaflutole & 75 & 71,9 & b & 20 & b & 1906 & a & 35,7 & $\mathrm{~b}$ \\
\hline CV (\%) & & 18,68 & & 12,44 & & 18,92 & & 23,18 & \\
\hline
\end{tabular}

Maiores decréscimos na taxa fotossintética (A) e na condutância estomática (gs) foram constatadas nas plantas de girassol onde os herbicidas fomesafen, a mistura (fluazifop-p-buthyl + fomesafen) e o clorimuron-ethyl foram aplicados, sendo que, os demais tratamentos não apresentaram diferenças em relação à testemunha (Tabela 5). Como o herbicida fomesafen pertence ao grupo dos inibidores da PPO, na rota responsável pela síntese de clorofila, seus danos à cultura podem ser diretamente aferidos pela mensuração da taxa fotossintética e de variáveis associadas a ela (Silva et al., 2007). Considerando o mecanismo de ação dos herbicidas inibidores da PPO, a atividade fotossintética é comprometida por alguns distúrbios relacionados com a peroxidação de lipídios e pigmentação foliar. Os herbicidas deste grupo atuam na redução dos teores de clorofila e carotenoides foliares, efeitos estes que podem ser observados também em plantas tolerantes (Carretero, 2008). Estes 
herbicidas podem atuar também na liberação de etileno, etano e aldeído malônico (Kenyon \& Duck, 1985), que podem atuar no balaço hormonal, e consequentemente em várias atividades fisiológicas das plantas.

A redução da condutância estomática foi observada em soja e Portulaca oleracea 6 horas após a aplicação do lactofen (Wichert \& Talbert, 1993). Este herbicida pode promover o fechamento estomático devido aos processos oxidativos e aumento da concentração de óxido nítrico, estes que atuam como sinalizadores para o ABA, hormônio que atua regulando o fechamento estomático (Webb et al., 2001). Esta atuação do óxido nítrico tem sido observada em plantas de soja após a aplicação de lactofen (Carretero, 2008).

Decréscimo nos valores da taxa transpiratória (E) foi observado quando as plantas de girassol foram submetidas ao tratamento com os herbicidas fomesafen, fluazifop-p-buthyl + fomesafen, fluazifop-pbuthyl, clethodim e chlorimuron-ethyl. Os demais tratamentos não diferiram das parcelas onde não foi aplicado qualquer produto (Tabela $5)$.

Plantas de girassol tratadas com fomesafen mostraram redução nos valores da relação carbono interno/carbono atmosférico $(\mathrm{Ci} / \mathrm{Ca})$ em relação à testemunha, entretanto, os herbicidas flumixazin, fenoxaprop-p-ethyl + clethodim, fluazifop-p-buthyl e clethodim apresentaram relação $\mathrm{Ci} / \mathrm{Ca}$ superiores aos observados na testemunha. Assim os demais produtos não diferiram da testemunha sem herbicidas (Tabela 5).

Tabela 5. Taxa de assimilação líquida de $\mathrm{CO}_{2}\left(\mathrm{~A}-\mu \mathrm{mol} \mathrm{CO}_{2} \mathrm{~m}^{-2} \mathrm{~s}^{-1}\right)$, condutância estomática de vapor d'água (gs - mmol $\mathrm{H}_{2} \mathrm{O} \mathrm{m}^{-2} \mathrm{~s}^{-1}$ ) taxa transpiratória $\left(\mathrm{E}-\mathrm{mmol} \mathrm{H}_{2} \mathrm{O} \mathrm{m}^{-2} \mathrm{~s}^{-1}\right.$ ), relação entre as concentrações interna e externa de $\mathrm{CO}_{2}\left(\mathrm{C}_{\mathrm{i}}: \mathrm{C}_{\mathrm{a}}\right)$, eficiência instantânea no uso da água (EUA - $\mu$ mol de $\mathrm{CO}_{2} \mathrm{mmol}^{-1}$ de $\left.\mathrm{H}_{2} \mathrm{O}\right)$ e consumo de $\mathrm{CO}_{2}(\Delta \mathrm{C})$ em folhas de girassol, 25 dias após a aplicação (DAH) de diferentes herbicidas. Viçosa (MG), 2013.

\begin{tabular}{|c|c|c|c|c|c|c|c|c|c|c|c|c|c|}
\hline Tratamentos & Dose $\left(\mathrm{g}\right.$ i.a. ha $\left.^{-1}\right)$ & A & & $g_{s}$ & & E & & $\mathrm{C}_{\mathrm{i}} / \mathrm{C}_{\mathrm{a}}$ & & EUA & & $\Delta \mathrm{C}$ & \\
\hline Testemunha ${ }^{1}$ & --- & 25,71 & a & 1,38 & $\mathrm{a}$ & 9,11 & $a$ & 0,88 & $\mathrm{~b}$ & 2,89 & $a$ & 35,32 & \\
\hline Flumioxazin & 50 & 20,90 & $\mathrm{a}$ & 1,58 & $\mathrm{a}$ & 9,68 & $\mathrm{a}$ & 0,91 & $\mathrm{a}$ & 2,17 & $\mathrm{a}$ & 29,79 & \\
\hline Fomesafen & 250 & 9,70 & b & 0,26 & $\mathrm{~b}$ & 3,99 & $\mathrm{~b}$ & 0,83 & $\mathrm{c}$ & 3,26 & $\mathrm{a}$ & 13,76 & b \\
\hline Lactofen & 180 & 25,72 & $\mathrm{a}$ & 1,13 & $\mathrm{a}$ & 7,54 & $\mathrm{a}$ & 0,87 & b & 3,72 & $\mathrm{a}$ & 34,75 & $\mathrm{a}$ \\
\hline Flu & $225+2$ & 11,43 & b & 0,53 & $\mathrm{~b}$ & 4,77 & $\mathrm{~b}$ & 0,88 & b & 2,48 & $\mathrm{a}$ & 16,37 & $\mathrm{~b}$ \\
\hline $\mathrm{Fe}$ & $93,75+9$ & 21,19 & $\mathrm{a}$ & 1,41 & $\mathrm{a}$ & 9,59 & $\mathrm{a}$ & 0,90 & $\mathrm{a}$ & 2,35 & $\mathrm{a}$ & 30,04 & $\mathrm{a}$ \\
\hline $\mathrm{Hal}$ & 60 & 27,45 & a & 1,47 & $\mathrm{a}$ & 8,33 & $\mathrm{a}$ & 0,89 & b & 3,34 & $\mathrm{a}$ & 37,22 & $\mathrm{a}$ \\
\hline p-butyl & 187,5 & 22,70 & $a$ & 1,54 & $\mathrm{a}$ & 7,02 & $\mathrm{~b}$ & 0,91 & $\mathrm{a}$ & 3,40 & $\mathrm{a}$ & 31,11 & $\mathrm{a}$ \\
\hline Clet & 96 & 23,41 & $\mathrm{a}$ & 1,75 & $\mathrm{a}$ & 6,30 & $\mathrm{~b}$ & 0,92 & $\mathrm{a}$ & 4,00 & $\mathrm{a}$ & 31,73 & $\mathrm{a}$ \\
\hline ron- $\epsilon$ & 20 & 13,77 & $\mathrm{~b}$ & 0,68 & $\mathrm{~b}$ & 5,80 & $\mathrm{~b}$ & 0,89 & $\mathrm{~b}$ & 2,65 & $\mathrm{a}$ & 19,63 & $\mathrm{~b}$ \\
\hline Isoxaflutole & 75 & 23,16 & $\mathrm{a}$ & 1,50 & $\mathrm{a}$ & 8,78 & $\mathrm{a}$ & 0,90 & $\mathrm{a}$ & 2,75 & $\mathrm{a}$ & 32,23 & $\mathrm{a}$ \\
\hline $\mathrm{CV}(\%)$ & & 24,2 & & 31,19 & & 23,85 & & 2,81 & & 44,21 & & 21,46 & \\
\hline
\end{tabular}

${ }^{1}$ Médias seguidas da mesma letra na coluna não diferem significativamente entre si, pelo teste Scott-Knott, ao nível de $5 \%$ de probabilidade.

Não foi observada diferença entre os tratamentos com relação à eficiência do uso da água (EUA), enquanto que para $\quad \mathrm{CO}_{2}$ consumido $(\Delta \mathrm{C})$, os herbicidas fomesafen, a mistura fluazifop-p-buthyl + fomesafen e o chlorimuron-ethyl promoveram decréscimo nos valores dessa variável, sendo que, os demais tratamentos não afetaram o $\Delta \mathrm{C}$ (Tabela 5). Segundo Galon et al. (2010), o $\Delta \mathrm{C}$ está diretamente relacionado à intensidade fotossintética da planta no momento da avaliação, ou seja, quanto mais lento for o metabolismo da planta, menor o consumo de $\mathrm{CO}_{2}$ por unidade de tempo, reduzindo a diferença entre a concentração de carbono da atmosfera e do espaço interno da folha $(\Delta C)$.

A taxa fotossintética está diretamente relacionada à radiação fotossinteticamente ativa, ou seja, ao comprimento de onda de luz de 400 a 700 nanômetros, espectro de radiação que 
está envolvido na fotossíntese, e indiretamente aos fatores relacionados, às trocas gasosas e disponibilidade hídrica (Naves-Barbiero et al., 2000), sendo altamente dependente da abertura estomática. Dessa forma, os herbicidas fomesafen, a mistura fluazifop-p-buthyl + fomesafen e o chlorimuron-ethyl promoveram a redução da gs e assim afetando negativamente de forma direta o consumo de $\mathrm{CO}_{2}$, a $\mathrm{A}$ e a $\mathrm{E}$ das plantas.

\section{Conclusões}

Os herbicidas testados interferem de forma variada nas plantas de girassol. Os herbicidas flumioxazin, fenoxaprop-p-ethyl + clethodim, haloxyfop-p-methyl, fluazifop-pbuthyl e clethodim mostram-se seletivos a cultura.

O fomesafen, a mistura fluazifop-pbuthyl + fomesafen e chlorimuron-ethyl afetam negativamente o crescimento e os parâmetros fotossintéticos (A, gs, E e $\Delta \mathrm{C})$ do girassol, sendo considerados não seletivos à cultura.

\section{Agradecimentos}

Os autores agradecem à Coordenação de Aperfeiçoamento e Pessoal de Nível Superior (CAPES) e à Fundação de Amparo a Pesquisa do Estado de Minas Gerais (FAPEMIG) pelo apoio financeiro e pelas bolsas concedidas.

\section{Referências}

BRASIL. Ministério da Agricultura, Pecuária e Abastecimento. Agrofit: consulta de produtos formulados. 2014. Disponível em: $<$ http://extranet.agricultura.gov.br/agrofit_c ons/principal_agrofit_cons $>$. Acesso em: 15 fev. 2014.

BRIGHENTI, A.M. et al. Persistência e fitotoxicidade de herbicidas aplicados na soja sobre o girassol em sucessão. Pesquisa Agropecuária Brasileira, v.37, n.4, p.559-565, 2002.
BRIGHENTI, A.M. et al. Cadastramento fitossociológico de plantas daninhas na cultura do girassol. Pesquisa Agropecuária Brasileira, v.38, n.5, p.651-657, 2003.

BRIGHENTI, A.M. et al. Períodos de interferência de plantas daninhas na cultura do girassol. Planta Daninha, v.22, n.2, p.251-257, 2004.

BRIGHENTI, A.M. Resistência do girassol a herbicidas inibidores da enzima acetolactatosintase. Pesquisa Agropecuária Tropical, v.42, n.2, p.225-230, 2012.

CARRETERO, D.M. Efeitos da inibição da protoporfirinogênio IX oxidase sobre as trocas gasosas e fluorescência da clorofila a em plantas de soja (Glycine max L. Merrill). 2008. 57f. Dissertação (Mestrado em Fisiologia Vegetal). Universidade Federal de Viçosa, Viçosa, 2008.

CASTRO, C. et al. A cultura do girassol. Londrina: Embrapa - CNPSo, 1997. 38p. (Embrapa-CNPSo. Circular Técnica, 13).

ELEZOVIC, I. et al. Yield and yield components of imadazolinone-resistant sunflower (Helianthus annuus L.) are influenced by pre-emergence herbicide and time of post-emergence weed removal. Field Crops Research, v.128, n.14, p.137-146, 2012.

GALON, L. et al. Influência de herbicidas na atividade fotossintética de genótipos de canade-açúcar. Planta Daninha, v.28, n.3, p.591597, 2010.

KENYON, W.H.; DUKE, S.O. Effects of acifluorfen on endogenous antioxidants and protective enzymes in cucumber (Cucumis sativus L.) cotyledons. Plant Physiology, v.79, n.3, p.862-866, 1985.

NAVES-BARBIERO, C.C. et al. Fluxo de seiva e condutância estomática de duas espécies lenhosas sempre-verdes no campo sujo e cerradão. Revista Brasileira de Fisiologia Vegetal, v.12, n.2, p.119-134, 2000. 
PANNACCI, E.; GRAZIANI, F.; COVARELLI, G. Use of herbicide mixtures for pre and post-emergence weed control in sunflower (Helianthus annuus). Crop Protection, v.26, n.8, p.1150-1157, 2007.

SILVA, A.A.; FERREIRA, F.A.; FERREIRA, L.R. Herbicidas: Classificação e mecanismo de ação. In: SILVA, A.A.; SILVA, J.F. (Eds.). Tópicos em manejo de plantas daninhas. Viçosa, MG: Universidade Federal de Viçosa, 2007. v.2, cap.3, p.58-117.

SILVA, D.V. et al. Seletividade de herbicidas pós-emergentes na cultura da mandioca. Planta Daninha, v.30, n.4, p.835-841, 2012.

SOCIEDADE BRASILEIRA DA CIÊNCIA DAS PLANTAS DANINHAS SBCPD. Procedimentos para instalação, avaliação e análise de experimentos com herbicidas. Londrina: 1995. 42p.

WEBB, A.A.R. et al. The role of calcium in ABA-induced gene expression and stomatal movements. Plant Journal, v.26, n.3, p.351362, 2001.

WICHERT, R.A.; TALBERT, R.E. Soybean (Glycine $\max$ L.) response to lactofen. Weed Science, v.41, n.3, p.23-27, 1993.

ZABLOTOWICZ, R.M.; REDDY, K.N. Nitrogenase activity, nitrogen content, and yield responses to glyphosate in glyphosate-resistant soybean. Crop Protection, v.26, n.3, p.370276, 2007. 